\title{
Mitigation of Insulin Resistance by Mangiferin in a Rat Model of Fructose-Induced Metabolic Syndrome Is Associated with Modulation of CD36 Redistribution in the Skeletal Muscle
}

\author{
Liang Zhou, Yongquan Pan, Ritsu Chonan, Robert Batey, Xianglu Rong, Johji Yamahara, \\ Jianwei Wang, and Yuhao Li \\ Faculty of Basic Medical Sciences (L.Z.), Laboratory Animal Center (Y.P.), and Laboratory of Traditional Chinese Medicine (J.W.), \\ Chongqing Medical University, Chongqing, China; Koei Kogyo Co., Ltd., Tokyo, Japan (R.C.); Central Clinical School, Royal \\ Prince Alfred Hospital, The University of Sydney, Sydney, Australia (R.B.); Guangdong Metabolic Diseases Research Center of \\ Integrated Chinese and Western Medicine, and the Institute of Chinese Medicine, Guangdong Pharmaceutical University, \\ Guangzhou, China (X.R.); Pharmafood Institute, Kyoto, Japan (J.Y.); and Endocrinology and Metabolism Group, Institute of \\ Health Sciences/Sydney Institute of Traditional Chinese Medicine, Sydney, Australia (Y.L.)
}

Received August 26, 2015; accepted October 22, 2015

\begin{abstract}
Mangiferin is one of the prominent active components responsible for the antidiabetic property of many traditional herbs, but its underlying mechanisms of action remain unclear. CD36 in skeletal muscle is known to contribute to the etiology of insulin resistance by facilitating fatty acid uptake. This study investigated the effect of mangiferin on insulin resistance. The results showed that treatment of Wistar-Kyoto rats with mangiferin (15 mg/kg, once daily, by oral gavage) for 7 weeks inhibited chronic liquid fructose consumption-induced increases in plasma insulin concentrations at the baseline and during oral glucose tolerance test (OGTT), and the homeostasis model assessment of insulin resistance index. It also suppressed the increases in fasted plasma nonesterified fatty acid (NEFA) concentration and the adipose tissue insulin resistance index. Mechanistically, mangiferin neither affected intakes of fructose and chow, and the increase in epididymal and
\end{abstract}

perirenal fat, nor attenuated fructose-induced hypertension. In contrast, mangiferin attenuated fructose-induced acceleration of plasma NEFA clearance during OGTT, and tended to decrease excessive triglyceride accumulation in gastrocnemius. Immunofluorescence staining and subsequent rating of CD36-expressing fibers in gastrocnemius revealed that mangiferin restored fructose-stimulated sarcolemmal CD36 overexpression and decreased intracellular CD36 distribution. In addition, the effects of mangiferin on the parameters associated with insulin resistance and abnormal fatty acid metabolism were absent in the spontaneously hypertensive rats carrying numerous nonfunctional mutations in the CD36 gene. Thus, these results suggest that mangiferin treatment mitigates insulin resistance in a rat model of fructose-induced metabolic syndrome by modulating sarcolemmal and intracellular CD36 redistribution in the skeletal muscle.

\section{Introduction}

The metabolic syndrome is a clustering of components that reflect overnutrition, sedentary lifestyles, and resultant excess adiposity. A striking increase in the number of people with the metabolic syndrome worldwide has taken place over the past several decades. The pathophysiology of the metabolic syndrome is largely attributable to insulin resistance with excessive flux of fatty acids (Eckel et al., 2005). Therefore,

This work was supported financially by grants from the National Natural Science Foundation of China, China [Grant No: 81374033]; Chongqing Science and Technology Commission, China [Grant No: CSTC2012gg-yyjsB10002-1]; the Natural Science Foundation of Chongqing Medical University, China (Grant No: 201418); and R\&D Agency for Curative Natural Products (a Japanese government-registered nonprofit organization), Kyoto, Japan. dx.doi.org/10.1124/jpet.115.229005 the metabolic syndrome is also called "the insulin resistance syndrome." Insulin resistance has traditionally been defined with a glucocentric view-i.e., when a defect in insulin action results in fasting hyperinsulinemia, or postprandial hyperinsulinemia exists even before fasting hyperinsulinemia develops (Eckel et al., 2005). Insulin resistance, hyperinsulinemia, dyslipidemia, and obesity precede the progression to type 2 diabetes in $75-85 \%$ of patients (Lebovitz, 1999). Additionally, hyperinsulinemia is the strongest predictor of diabetes incidence (Hanson et al., 2002).

Mangiferin, a xanthone glucoside, is one of the prominent active components contained in many traditional antidiabetic herbs, such as Anemarrhena asphodeloides, Mangifera indica, and Salacia species. Mangiferin and the related herbs have been demonstrated to have antidiabetic activity in rodents (Ichiki et al., 1998; Li et al., 2004; Li et al., 2008; Muruganandan et al., 2005;

ABBREVIATIONS: Adipo-IR, adipose tissue insulin resistance; AUC, the area under the curve; e+p FAT, epididymal and perirenal fat; HOMA-IR, homeostasis model assessment of insulin resistance; MCP, monocyte chemoattractant protein; NEFA, nonesterified fatty acids; OGTT, oral glucose tolerance test; PCR, polymerase chain reaction; PPAR, peroxisome proliferator-activated receptor; SBP, systolic blood pressure; SHR, spontaneously hypertensive rats; TNF, tumor necrosis factor; WKY, Wistar-Kyoto rats. 
Akase et al., 2011; Apontes et al., 2014; Han et al., 2015). Furthermore, it has been recently reported that mangiferin supplementation improves insulin resistance index in overweight patients (Na et al., 2015). However, the underlying mechanisms of action are still largely unknown. We and others have reported that they improve numerous lipid metabolism-associated abnormalities, such as fatty liver, hyperlipidemia, excessive cardiac lipid accumulation and obesity (Huang et al., 2006a,b; Guo et al., 2011; Niu et al., 2012; Liu et al., 2013; Xing et al., 2014; Na et al., 2015). We hypothesized that regulation of lipid metabolism might be associated with the antidiabetic property of mangiferin.

Long-chain fatty acids are common dietary nutrients, not only a major energy source for most cells and precursors for synthesis of cellular lipids with structural or signaling functions, but also a regulator of gene expression via transcription factors (Su and Abumrad, 2009). Membrane uptake of long-chain fatty acids is the first step in cellular fatty acid utilization and a point of metabolic regulation. CD36/fatty acid transporter is a multifunctional glycoprotein with particular abundance in adipose tissue, skeletal muscle, and heart, but with low expression in kidneys and liver; CD36 facilitates a major fraction of fatty acid uptake by the key tissues, and is associated with membrane transport and utilization of longchain fatty acids (Su and Abumrad, 2009). Studies in rodents implicate CD36 in a number of metabolic pathways with relevance to obesity and its associated complications (LoveGregory and Abumrad, 2011). Research findings indicate that CD36 overexpression predisposes to metabolic complications, whereas lower CD36 expression is metabolically protective (Love-Gregory et al., 2011). CD36 dysfunction might contribute to development of insulin resistance and the metabolic syndrome (Su and Abumrad, 2009). Thus, CD36 might provide a useful therapeutic target for the prevention and/or treatment of insulin resistance.

Strong evidence suggests that chronically high consumption of fructose in rodents results in the metabolic syndrome and other metabolic abnormalities (Tappy and Lê K, 2010). Recent clinical findings have also demonstrated that dietary fructose increases hepatic de novo lipogenesis, promotes dyslipidemia, decreases insulin sensitivity, and increases visceral adiposity in overweight/obese adults (Stanhope et al., 2009). In the present study, we examined the effect of mangiferin on insulin resistance in a rat model of metabolic syndrome induced by chronic fructose consumption, and further investigated the possible involvement of CD36 in skeletal muscle that is prominently associated with fatty acid uptake.

\section{Materials and Methods}

\section{Animals, Diet, and Experimental Protocol}

All animal procedures were in accordance with the Principles of Laboratory Animal Care (http://grants1.nih.gov/grants/olaw/references/ phspol.htm) and were approved by the Animal Ethics Committee, Chongqing Medical University, Chongqing, China.

Male Wistar-Kyoto rats (WKY) and the spontaneously hypertensive rats (SHR)/NCrl weighing 230-250 g were purchased from Beijing Vital River Laboratory Animal Technology Co., Ltd. (Beijing, China) and the standard diet was supplied by the laboratory animal center of Chongqing Medical University. Rats were housed under specific pathogen-free conditions in a temperature- and humidity-controlled facility $\left(21 \pm 1^{\circ} \mathrm{C}, 55 \pm 5 \%\right.$ relative humidity) with a 12-hour light/ dark cycle. Animals were allowed free access to water and the standard diet for at least 1 week prior to the start of the experiments.

We have recently demonstrated that treatment of fructose-fed SHR with mangiferin at $15 \mathrm{mg} / \mathrm{kg}$, but not $5 \mathrm{mg} / \mathrm{kg}$, showed a significant antisteatotic effect (Xing et al., 2014). Therefore, the dosage of $15 \mathrm{mg} / \mathrm{kg}$ was chosen. Two experiments were performed as follows.

Experiment in WKY. Eighteen WKY were divided into three groups ( $n=6$ per group): 1 ) WKY water control, free access to water; 2 ) WKY fructose control, free access to $10 \%$ fructose solution (w/v, preparation every day); 3) WKY fructose + mangiferin. Animals in mangiferin-treated groups were administered mangiferin $15 \mathrm{mg} / \mathrm{kg}$ (suspended in 5\% gum arabic solution, gavage once daily; SigmaAldrich, Chongqing, China) for 7 weeks. The rats in the corresponding water- and fructose-control groups received vehicle (5\% gum arabic) alone. Systolic blood pressure (SBP) was measured in conscious rats by a tail-cuff method (Model MK-2000ST; Muromachi Kikai Co. Ltd, Tokyo, Japan) prior to and 6 weeks after the experiment commenced. At least six readings were taken for each measurement. All rats had free access to the standard chow. To avoid stress and maintain accurate monitoring of fructose intake, only two rats were housed in a cage at any given time. The consumed chow and fructose solution were measured per two rats daily, and the intake of fructose was calculated. At the end of week 6 , blood samples were collected by retroorbital venous puncture under ether anesthesia after overnight fast. Here, the plasma concentrations of glucose (kit from Kexin Institute of Biotechnology, Shanghai, China), insulin (kit from Morinaga Biochemical Industries, Tokyo, Japan), triglyceride (Triglyceride-E kit; Wako, Osaka, Japan), and nonesterified fatty acids (NEFA) (NEFA-C kit; Wako) were determined using enzymatic methods or by enzymelinked immunosorbent assay. Immediately following, oral glucose tolerance tests (OGTT) were performed. Animals were weighed and euthanized after being fasted overnight at the end of week 7 . Epididymal and perirenal fat $(e+p$ FAT $)$ were collected and weighed. Segments of gastrocnemius and liver were individually snap frozen in liquid nitrogen and stored at $-80^{\circ} \mathrm{C}$ for subsequent determination of gene expression and/or the content of triglyceride.

TABLE 1

Primer sequences for real time PCR assays

\begin{tabular}{|c|c|c|}
\hline Gene & Accession Number & Primer Sequences \\
\hline$\beta$-actin & NM_031144.2 & $\begin{array}{l}\text { Forward: ACGGTCAGGTCATCACTATCG } \\
\text { Reverse: GGCATAGAGGTCTTTACGGATG }\end{array}$ \\
\hline Adiponectin & NM_144744.3 & $\begin{array}{l}\text { Forward: cgttctcttcacctacgaccagt } \\
\text { Reverse: attgttgtcccettccccatac }\end{array}$ \\
\hline CD36 & NM_001109218 & $\begin{array}{l}\text { Forward: AACCCAGAGGAAGTGGCAAAG } \\
\text { Reverse: GACAGTGAAGGCTCAAAGATGG }\end{array}$ \\
\hline MCP-1 & AF079313.1 & $\begin{array}{l}\text { Forward: CGGTTTCTCCCTTCTACTTCCTG } \\
\text { Reverse: GCTCTGCCTCAGCCTTTTATTG }\end{array}$ \\
\hline PPAR- $\gamma$ & AB011365.1 & $\begin{array}{l}\text { Forward: GCCCTTTGGTGACTTTATGGAG } \\
\text { Reverse: GCAGCAGGTTGTCTTGGATGT }\end{array}$ \\
\hline TNF- $\alpha$ & NM_012675.3 & $\begin{array}{l}\text { Forward: ATGGGCTCCCTCTCATCAGTTC } \\
\text { Reverse: CTCCTCCGCTTGGTGGTTTG }\end{array}$ \\
\hline
\end{tabular}


TABLE 2

General information in WKY

\begin{tabular}{lccc}
\hline \multicolumn{1}{c}{ Parameter } & Water Control & Fructose Control & Fructose Mangiferin \\
\hline SBP before $(\mathrm{mmHg})$ & $128.3 \pm 1.2$ & $130.5 \pm 1.3$ & $130.0 \pm 2.0$ \\
SBP after $(\mathrm{mmHg})$ & $131.7 \pm 2.2^{*}$ & $148.2 \pm 4.3$ & $144.0 \pm 4.3$ \\
Fructose intake $(\mathrm{g} / 7 \mathrm{w})$ & - & $997 \pm 72$ & $966 \pm 82$ \\
Chow intake $(\mathrm{g} / 7 \mathrm{w})$ & $1898 \pm 84^{*}$ & $941 \pm 41$ & $969 \pm 51$ \\
Body weight $(\mathrm{g})$ & $322.9 \pm 3.5$ & $306.8 \pm 2.3$ & $296.8 \pm 4.8$ \\
e + p FAT $(\mathrm{g})$ & $7.1 \pm 0.4^{*}$ & $8.0 \pm 0.4$ & $8.1 \pm 0.6$ \\
Plasma triglyceride $(\mathrm{mg} / \mathrm{dl})$ & $51.4 \pm 5.3^{*}$ & $74.5 \pm 2.8$ & $65.1 \pm 3.2$ \\
Hepatic triglyceride $(\mathrm{mg} / \mathrm{g}$ tissue $)$ & $16.9 \pm 1.6^{*}$ & $34.6 \pm 1.3$ & $26.8 \pm 2.5^{*}$ \\
\hline
\end{tabular}

Experiment in SHR. Eighteen SHR were also divided into three groups as WKY. The protocol was the same as that in WKY.

\section{OGTT}

After being fasted overnight, all rats received a glucose solution $(2 \mathrm{~g} / \mathrm{kg}$ in $5 \mathrm{ml}$ ) by the oral gavage. Blood samples were collected prior to and 20 , 60 , and 120 minutes after administration of glucose solution. Plasma concentrations of glucose, insulin, triglyceride and NEFA were determined. The homeostasis model assessment of insulin resistance (HOMAIR) index and the adipose tissue insulin resistance (Adipo-IR) index were calculated according to the following formulas, respectively: HOMA-IR index $=[$ fasted insulin $(\mu \mathrm{IU} / \mathrm{ml}) \times$ fasted glucose $(\mathrm{mM})] / 22.5$; Adipo-IR index $=$ fasted insulin $(\mathrm{mmol} / \mathrm{l}) \times$ fasted NEFA $(\mathrm{pmol} / \mathrm{l})($ Gastaldelli et al., 2009; Neuschwander-Tetri, 2010). The clearance of plasma NEFA was calculated: the concentration under fasted condition ( 0 minutes) - the concentration at the time-point $(20,60$, or 120 minutes) after glucose administration. The area under the curve (AUC) of plasma concentrations and/or clearances of glucose, insulin, or NEFA was calculated.

\section{Determination of Triglyceride Content in Liver and Skeletal Muscle}

Triglyceride content in liver and gastrocnemius was determined as described previously (Liu et al., 2013; Xing et al., 2014). Briefly, 100 $\mathrm{mg}$ of tissue was homogenized and extracted with $2 \mathrm{ml}$ of isopropanol. After centrifugation (3000 rpm), the triglyceride content in supernatants was determined enzymatically (Wako).

\section{Real-Time Polymerase Chain Reaction}

Real-time polymerase chain reaction (PCR) was performed as described previously (Xing et al., 2014). Total RNA was isolated from gastrocnemius of individual rats using TRIzol (Takara, Dalian, China). cDNA was synthesized using MMLV Reverse Transcriptase cDNA synthesis kit (Takara/Clontech, Dalian, China) according to the manufacturer's instructions. Real-time PCR was performed with the CFX 96 Real-Time PCR Detection System (Bio-Rad Laboratories Inc., Hercules, CA) using the SYBR Premix Ex Taq II (Takara/Clontech). The sequences of primers are shown in Table 1 . The gene expression
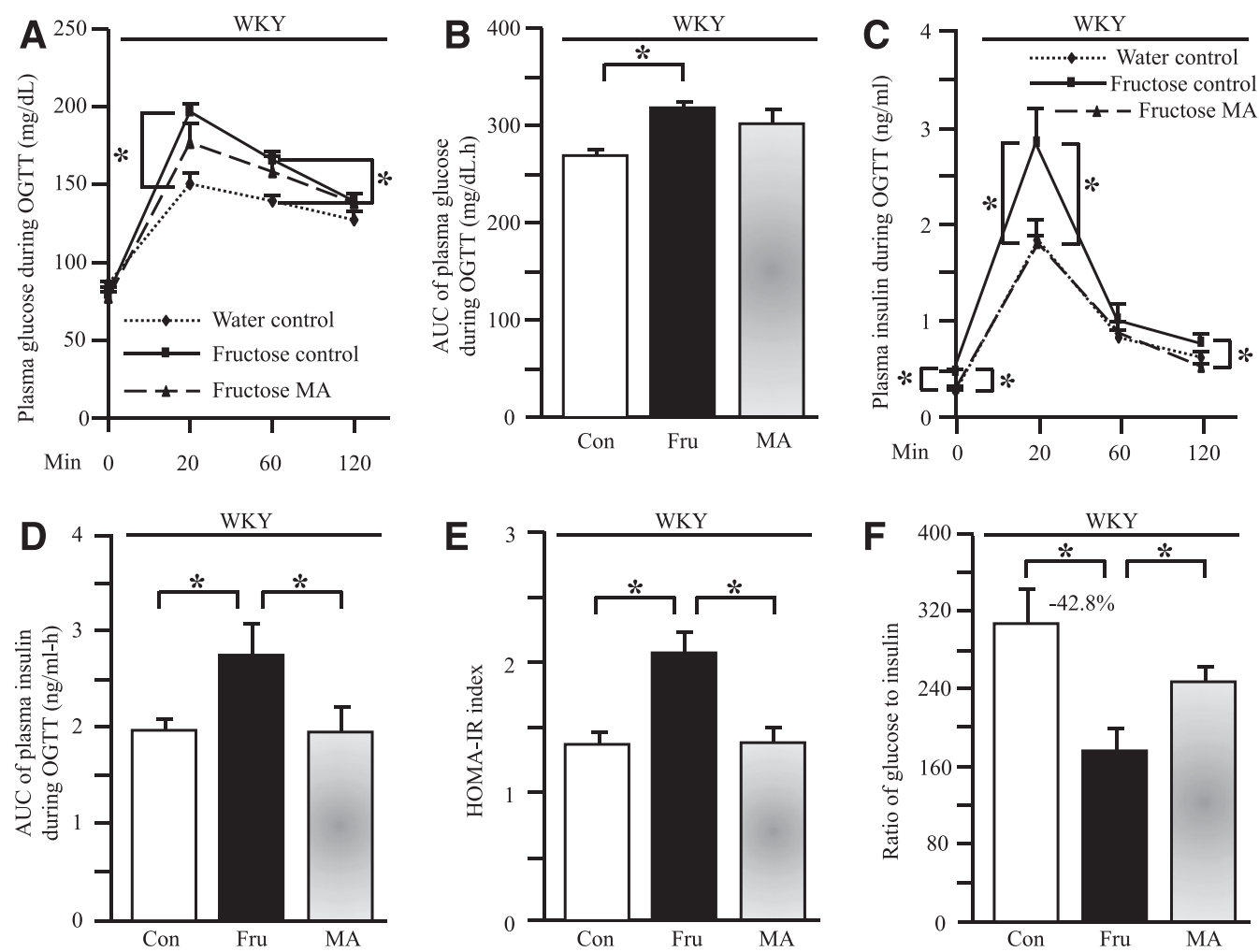

Fig. 1. Plasma glucose (A) and insulin (C) concentrations, and the AUC of glucose (B) and insulin (D) concentrations during OGTT, the HOMA-IR index (E), and the ratio of glucose to insulin (F) in WKY. The fructose controls (Fru) had free access to $10 \%$ fructose in their drinking water for 6 weeks, and the consumption of fructose in the mangiferin (MA) $(15 \mathrm{mg} / \mathrm{kg}$ )-treated (by gavage daily) rats was adjusted to match that of the fructose controls. The water controls (Con) had free access to a tap water. Data are means \pm S.E.M. $(n=6$ each group) versus control, $* P<0.05$. 

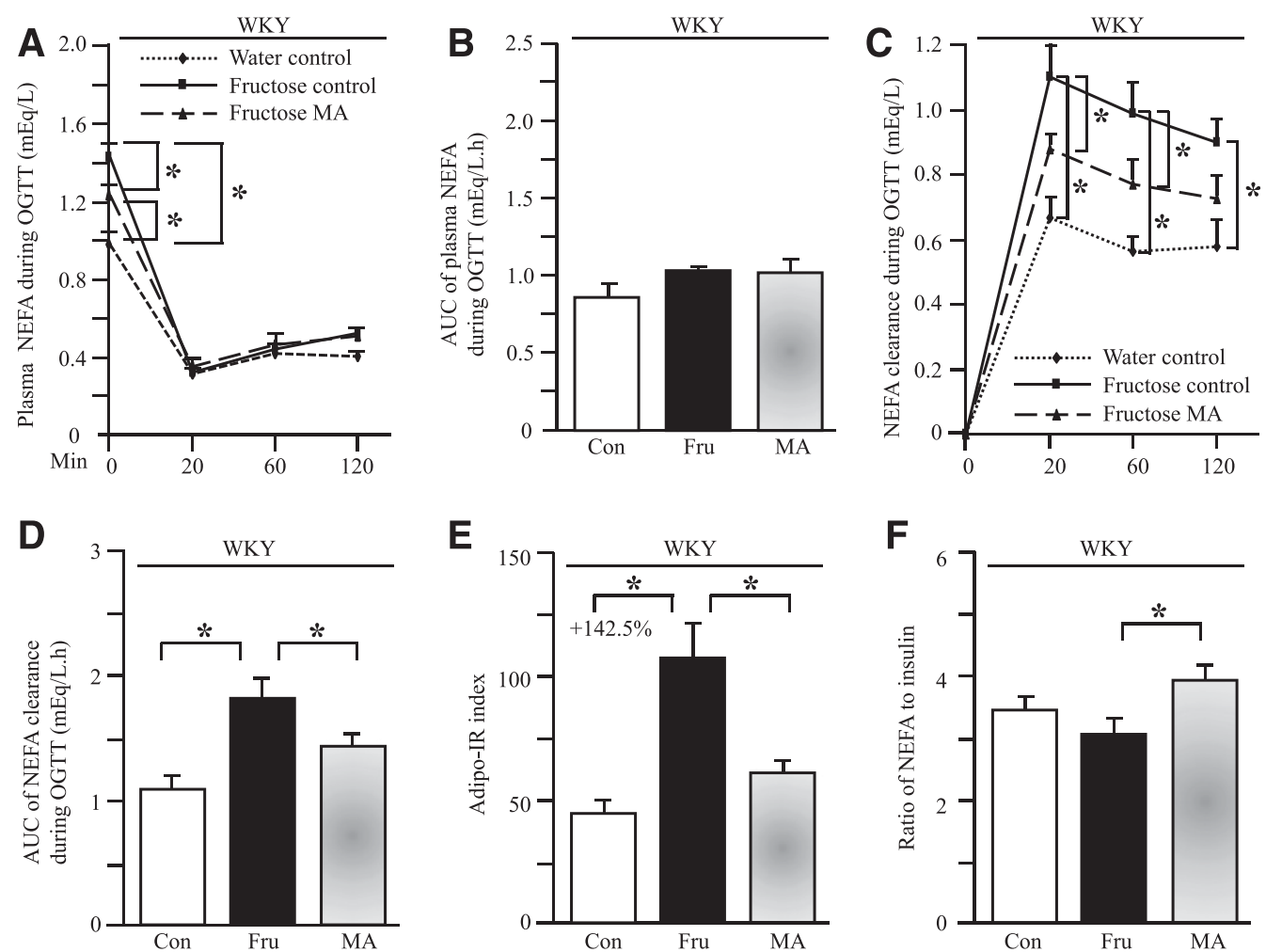

Fig. 2. Plasma NEFA concentrations (A), the AUC of plasma NEFA concentrations (B), plasma NEFA clearance (C), and the AUC of plasma NEFA clearance (D) during OGTT, the adipose tissue insulin resistance (Adipo-IR) index (E), and the ratio of NEFA to insulin (F) in WKY. The fructose controls (Fru) had free access to 10\% fructose in their drinking water for 6 weeks, and the consumption of fructose in the mangiferin (MA) (15 mg/kg)-treated (by gavage daily) rats was adjusted to match that of the fructose controls. The water controls (Con) had free access to a tap water. Data are means \pm S.E.M. $(n=6$ each group) versus control, $* P<0.05$.

from each sample was analyzed in duplicate and normalized against the internal control gene $\beta$-actin. Levels in control rats were arbitrarily assigned a value of 1 .

\section{Western Blot}

Western blot was performed basically as described previously (Liu et al., 2013; Xing et al., 2014). Total protein was prepared from gastrocnemius using the T-PER Tissue Protein Extraction Reagent kits (ThermoFisher Scientific, Sunnyvale, CA), according to the manufacturer's instructions. Protein concentration was determined using the Bradford method (Bio-Rad) using bovine serum albumin as a standard. Protein $(30 \mu \mathrm{g})$ was subjected to SDS-PAGE analysis on a $10 \%$ gel, then electrotransferred onto polyvinylidene fluoride membrane (Amersham/GE Healthcare UK Ltd., Buckinghamshire, UK). CD36 (dilution 1:1000; Abcam, Cambridge, MA) was detected with a rabbit polyclonal antibody. Detection of signal was performed using the ECL Western blot detection kit (Pierce/ThermoScientific) with antirabbit horseradish peroxidase-conjugated IgG (dilution 1:5,000; Santa Cruz Biotechnology, Dallas, TX) as second antibody, respectively. Polyclonal rabbit $\beta$-actin antibody (Cell Signaling Technologies, Beverly, MA) was used as loading control to normalize the signal obtained for CD36 protein. The immunoreactive bands were visualized by autoradiography and the density was evaluated using ImageJ 1.43. Levels in control rats were arbitrarily assigned a value of 1 .

\section{Immunofluorescence Staining}

Transverse cryosections from gastrocnemius were transferred to glass slides and allowed to dry at room temperature. The sections were blocked with normal goat serum for 30 minutes at room temperature and incubated with rabbit polyclonal anti-CD36 antibody (dilution 1: 200; Abcam, Cambridge, MA) in blocking buffer at $4^{\circ} \mathrm{C}$ overnight.
Sections were rinsed three times with phosphate-buffered saline and incubated with CY3-labeled goat anti-rabbit IgG as secondary antibody in blocking buffer for 30 minutes at room temperature. Sections were rinsed three times with phosphate-buffered saline again and nuclei were counterstained with DAPI (Molecular Probes/ ThermoFisher Scientific). Finally, sections were mounted and analyzed as described previously (Vistisen et al., 2004). Confocal images were collected with confocal microscope (A1R+ confocal microscope; Nikon Corporation, Tokyo, Japan). Imaging settings were set so that no signal was detected in the respective negative controls and a low fraction of pixels showed saturation intensity values when the stained samples were imaged. All images shown are representative of all six subjects.

\section{Rating of CD36-Expressing Fibers in Gastrocnemius}

The intensity of CD36 expression in the fibers of gastrocnemius was rated as described previously (Vistisen et al., 2004). On transverse cryosections from gastrocnemius stained with anti-CD36, the intensity of the fluorescent signal was rated $(1,2$, or 3$)$ by a person who was blinded. To check interobserver variability, another blinded person also rated the intensity of the fluorescence signal with similar results. In each sample, 100 fibers were rated. The percentage of fibers within each sample rated 1,2 , and 3 was calculated.

\section{Data Analysis}

All results with the exception of those for immunofluorescence are expressed as mean \pm S.E.M. Data were analyzed by ANOVA using StatView, and followed by Student-Newman-Keuls testing to locate the differences between groups. $P<0.05$ was considered to be statistically significant. 

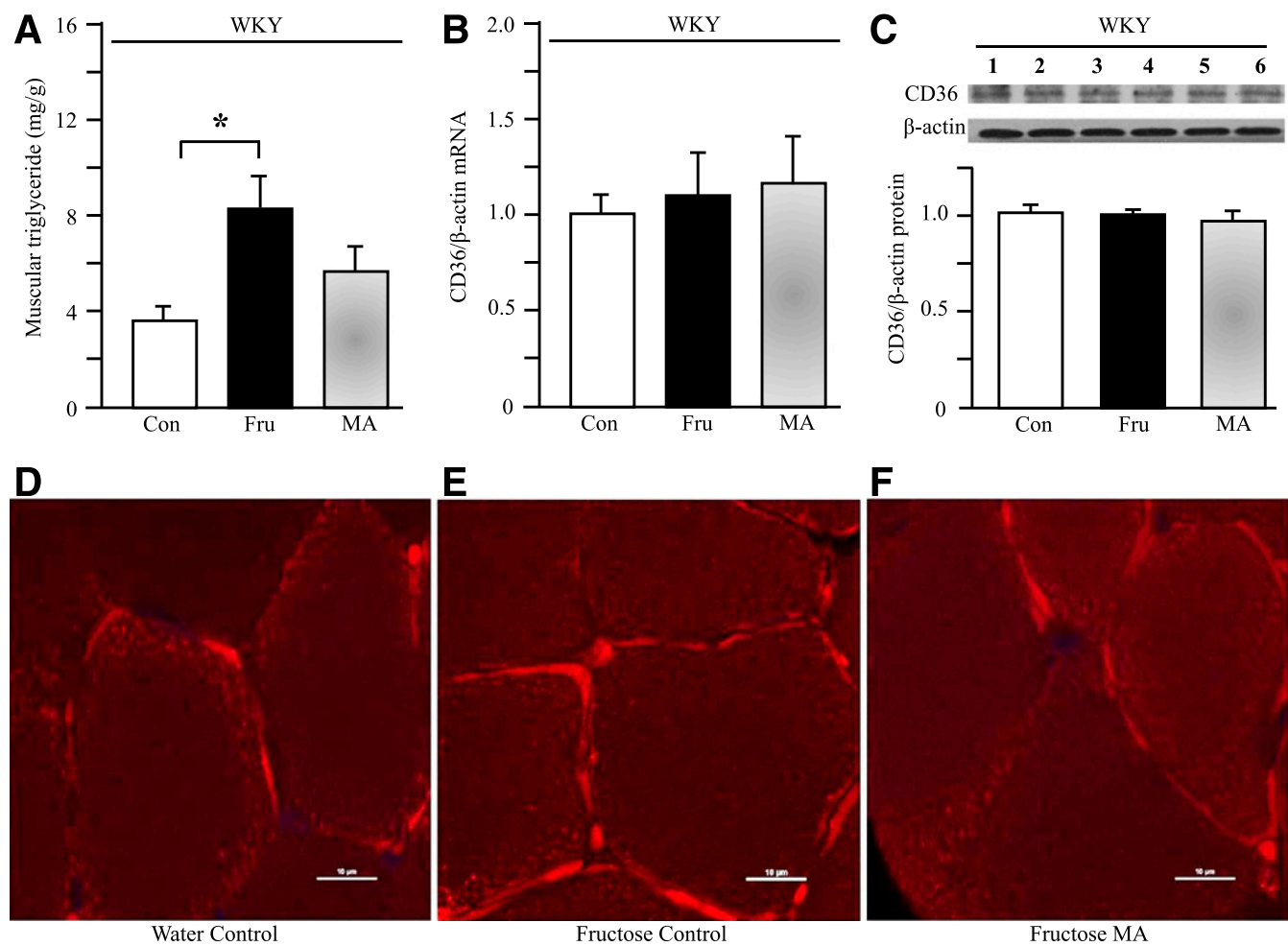

Fig. 3. Triglyceride content (A), expression of CD36 mRNA(B), and protein (C, Lane 1 and 2, water control; Lanes 3 and 4, fructose control; Lanes 5 and 6 , fructose mangiferin), and the CD36 expression by immunofluorescence staining (D-F) in the gastrocnemius of WKY. The fructose controls (Fru) had free access to $10 \%$ fructose in their drinking water over 7 weeks, and the consumption of fructose in the mangiferin (MA) (15 mg/kg)-treated (by gavage daily) rats was adjusted to match that of the fructose controls. The water controls (Con) had free access to a tap water. The methods for determination of mRNA expression by real-time PCR and protein expression by Western blot or immunofluorescence staining were described in Materials and Methods. Data are means \pm S.E.M. $(n=6$ each group) versus control, $* P<0.05$.

\section{Results}

General Information in WKY. Long-term fructose feeding increased SBP but decreased chow intake (Table 2). Although fructose feeding did not affect body weight, it significantly increased e+p FAT weight (by 12.7\%) (Table 2). Treatment with mangiferin did not affect SBP, intakes of fructose and chow, body weight, and $\mathrm{e}+\mathrm{p}$ FAT weight in fructose-fed rats (Table 2).

Effect on Glucose Metabolism in WKY. Under fasted condition, there was no significant difference in plasma glucose concentration between the three groups (Fig. 1A). However, fructose consumption increased plasma glucose concentrations at 20 minutes and the AUC of glucose concentrations during OGTT (Fig. 1, A and B). Fructose feeding increased plasma insulin concentrations at the baseline $(0$ minutes), 20, and 120 minutes after oral glucose challenge, the AUC of insulin concentrations during OGTT, and the HOMAIR index (Fig. 1, C-E). The ratio of glucose to insulin was also decreased by $42.8 \%$ (Fig. $1 \mathrm{~F}$ ). Although mangiferin treatment did not affect glucose concentrations in fructose-fed rats (Fig. $1, A$ and B), it suppressed fructose-induced increases in insulin concentrations under fasted condition and 20 minutes after oral glucose feeding (Fig. 1C), reduced the AUC of insulin concentrations during OGTT (Fig. 1D), and lowered the HOMA-IR index (Fig. 1E) in WKY. The decreased ratio of glucose to insulin was also restored (Fig. 1F).

Effect on Lipid Metabolism in WKY. Consistent with the previous findings (Tappy and Lê K, 2010; Xing et al., 2014), fructose feeding increased hepatic triglyceride content
(Table 2) and fasted plasma triglyceride concentrations (Table 2). Treatment with mangiferin at $15 \mathrm{mg} / \mathrm{kg}$ diminished fructose-induced hepatic triglyceride accumulation but did not restore the normal level (Table 2). Mangiferin treatment was without significant effect on fasted plasma triglyceride concentration (Table 2).

Also under fasted condition, plasma NEFA concentration (Fig. 2A) and the Adipo-IR index (increased by $142.5 \%$, Fig. $2 \mathrm{E}$ ) were higher in fructose-fed controls than in water controls. However, the difference disappeared at 20,60, and 120 minutes after oral glucose administration (Fig. 2A). Furthermore, there was no difference in the AUC of NEFA concentrations during OGTT between fructose-fed controls and water controls (Fig. 2B). Interestingly, the clearance of plasma NEFA (the decreased NEFA concentrations at different timepoints from the fasted condition) (Fig. 2C) and the AUC of NEFA clearance during OGTT (Fig. 2D) were higher in fructose controls than water controls. Fructose feeding also had tended to decrease the ratio of NEFA to insulin (Fig. 2F). Mangiferin treatment decreased fasted plasma NEFA concentration (Fig. 2A) and the Adipo-IR index (Fig. 2E), and increased the ratio of NEFA to insulin (Fig. 2F) in fructosefed rats. However, it did not affect NEFA concentrations at 20,60, and 120 minutes after oral glucose administration (Fig. 2A) and the AUC of NEFA concentrations during OGTT (Fig. 2B).

Fructose feeding significantly increased triglyceride content in gastrocnemius (Fig. 3A). Treatment with mangiferin tended to reduce the increase (Fig. $3 \mathrm{~A}, P>0.05$ ). 


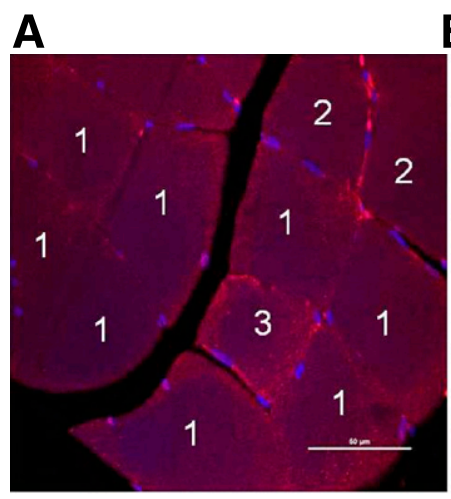

Water Control

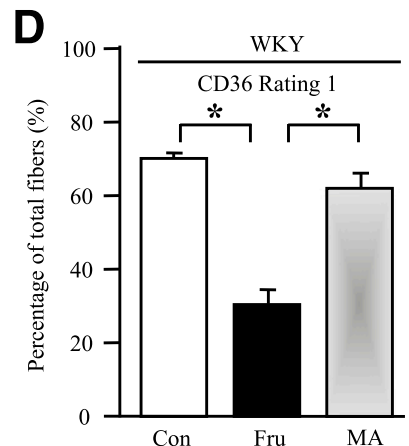

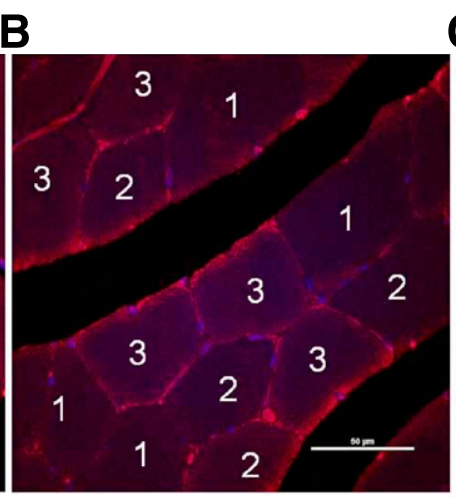

Fructose Control

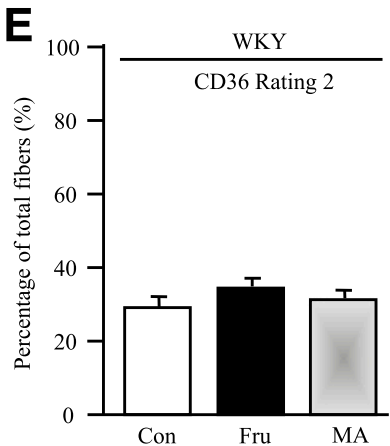

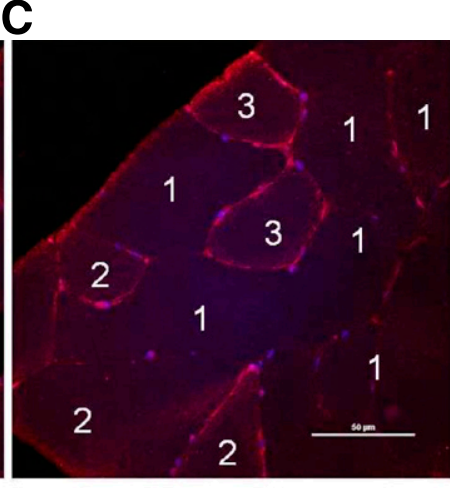

Fructose MA

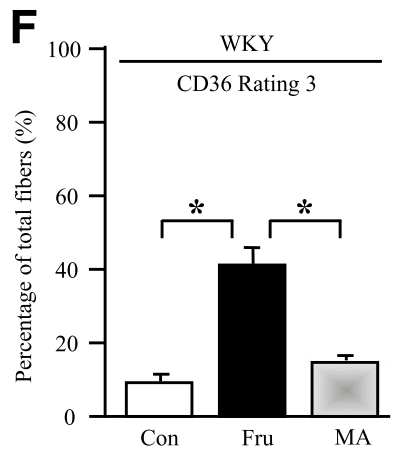

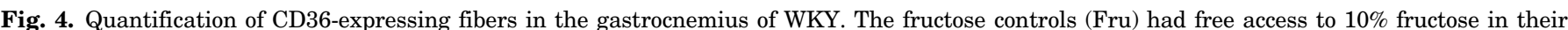

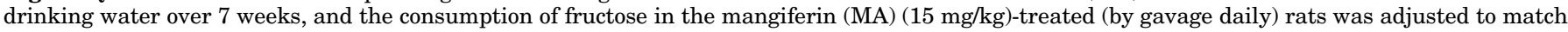

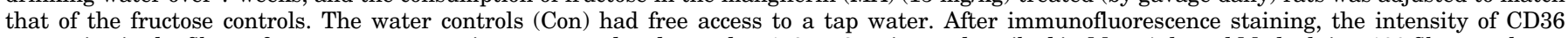

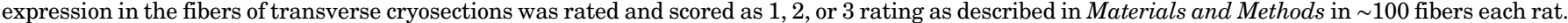

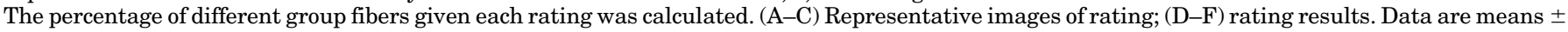
S.E.M. ( $n=6$ each group) versus control $* P<0.05$.

Muscular Gene/Protein Expression Profile in WKY. By real time PCR and Western blot, fructose feeding did not affect total muscular expression of CD36 mRNA and protein (Fig. 3, B and C). However, immunofluorescence staining showed that fructose feeding enhanced sarcolemma CD36 expression, whereas it decreased intracellular CD36 distribution (a fine punctate pattern) in gastrocnemius, compared with water control (Fig. 3, D and E). The results of further quantification of CD36-expressing fibers in gastrocnemius showed that fructose feeding decreased the percentage of fibers expressing sarcolemmal CD36 weakly (rating 1) but increased the percentage of fibers expressing sarcolemmal CD36 abundantly (rating 3), whereas it did not alter the percentage of fibers rated 2 (Fig. 4, A, B, and D-F). Mangiferin treatment reversed fructose-induced redistribution of CD36 in the skeletal muscle fibers (Figs. 3E and 4, C-F).

Also in skeletal muscle, fructose feeding did not induce any change in mRNA expression of peroxisome proliferatoractivated receptor (PPAR)- $\gamma$ (Fig. 5A), adiponectin (Fig. 5B), monocyte chemoattractant protein (MCP-1) (Fig. 5C), or tumor necrosis factor (TNF)- $\alpha$ (Fig. 5D). Mangiferin treatment also did not alter the expression of these genes in fructose-fed WKY (Fig. 5, A-D).

Effects in SHR. Consistent with the well-known characteristic, SHR were hypertensive (Table 3), compared with WKY (Table 2). The effects of mangiferin treatment on SBP, intakes of fructose and chow, body weight, $\mathrm{e}+\mathrm{p}$ fat weight, fasted plasma triglyceride concentration, hepatic triglyceride content, fasted plasma glucose concentration, and the AUC of glucose concentrations during OGTT in SHR (Table 3, Fig. 6, A and B) were similar to those in WKY (Table 2, Fig. 1, A and B).

Unlike in WKY, however, mangiferin did not affect plasma insulin concentrations at the baseline ( 0 minutes) and after oral glucose challenge, nor the AUC of insulin concentrations during OGTT, the HOMA-IR index, or the ratio of glucose to insulin (Fig. 6, C-F) in SHR. Moreover, mangiferin was without significant effect on the increases in fasted plasma NEFA concentration (Fig. 7A), the accelerated plasma NEFA clearance after oral glucose administration (Fig. 7C), the upregulated Adipo-IR index (Fig. 7E), and the ratio of NEFA to insulin (Fig. 7F).

As SHR carries a nonfunctional mutation in the CD36 gene (Aitman et al., 1999; Hajri et al., 2001), the gene expression in SHR was not analyzed in the present study.

\section{Discussion}

Hypertension (Ferrannini et al., 1987), caloric intake, and fat mass (Eckel et al., 2010) are associated with insulin resistance. The present study demonstrated that treatment of WKY with mangiferin suppressed fructose-induced increases in plasma insulin concentrations under fasted condition and after oral glucose administration, and restored the decreased ratio of glucose to insulin and the increased HOMA-IR index. Furthermore, mangiferin also decreased fasted plasma NEFA concentration, inhibited the increased Adipo-IR index, and upregulated the ratio of NEFA to insulin concentrations. As mangiferin neither attenuated fructose-induced hypertension nor affected 

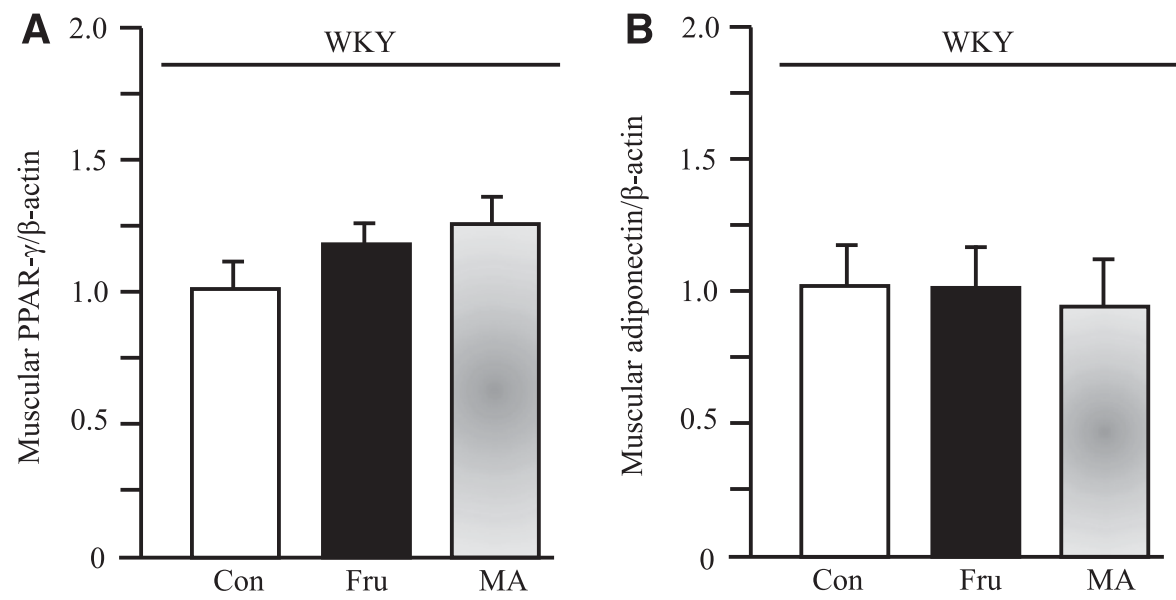

Fig. 5. Expression of peroxisome proliferatoractivated receptor (PPAR)- $\gamma(\mathrm{A})$, adiponectin (B), monocyte chemoattractant protein (MCP) (C), and tumor necrosis factor (TNF)- $\alpha$ (D) mRNAs in the gastrocnemius of WKY. The fructose controls (Fru) had free access to $10 \%$ fructose in their drinking water over 7 weeks, and the consumption of fructose in the mangiferin (MA)
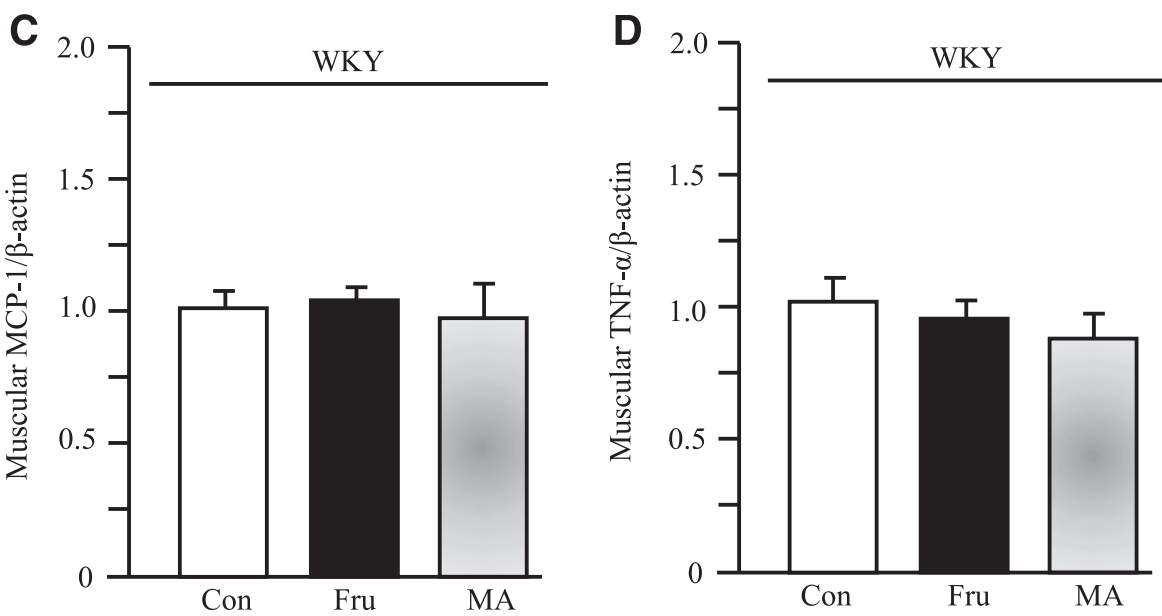
(15 $\mathrm{mg} / \mathrm{kg}$ )-treated (by gavage daily) rats was adjusted to match that of the fructose controls. The water controls (Con) had free access to a tap water. The method for determination of mRNA expression by real-time PCR was described in Materials and Methods. Data are means \pm S.E.M. ( $n=6$ each group).

intakes of fructose and chow and the increase in epididymal and perirenal fat, it appears that mangiferin treatment moderates insulin resistance via other distinct mechanisms.

A major contributor to the development of insulin resistance is an overabundance of circulating fatty acids (Eckel et al., 2005). In the setting of insulin resistance, insulin is unable to properly suppress lipolysis, resulting in increased flux of fatty acids from adipose tissue to nonadipose tissues, thereby further augmenting insulin resistance (Lewis et al., 2002; Cornier et al., 2008). After glucose stimulation, the strikingly increased plasma insulin concentration inhibits fatty acid release from adipose tissue. Thus, the clearance of plasma fatty acids during OGTT reflects the status of fatty acid uptake by such key tissues as skeletal muscle, liver, and heart. In the present study, fructose-induced insulin resistance was accompanied by acceleration of clearance of plasma NEFA during OGTT in WKY. Treatment with mangiferin suppressed the acceleration. These results suggest that mangiferin-elicited improvement of insulin resistance may be associated with fatty acid uptake by tissues.

Skeletal muscle is regarded as the major site of insulin resistance in obesity and type 2 diabetes (Kelley et al., 2002). Mounting evidence indicates that insulin resistance is highly associated with excessive intramyocellular triglyceride accumulation (Machann et al., 2004). During obesity an increase in the circulating fatty acids leads to increase in their uptake by

TABLE 3

General information in SHR

\begin{tabular}{lccc}
\hline Parameter & Water Control & Fructose Control & Fructose Mangiferin \\
\hline SBP before $(\mathrm{mmHg})$ & $185.2 \pm 2.6$ & $182.0 \pm 3.0$ & $189.5 \pm 3.9$ \\
SBP after $(\mathrm{mmHg})$ & $203.1 \pm 3.0$ & $197.2 \pm 5.2$ & $194.7 \pm 5.1$ \\
Fructose intake $(\mathrm{g} / 7 \mathrm{w})$ & - & $1015 \pm 61$ & $1043 \pm 45$ \\
Chow intake $(\mathrm{g} / 7 \mathrm{w})$ & $2187 \pm 59^{*}$ & $1253 \pm 60$ & $1214 \pm 18$ \\
Body weight $(\mathrm{g})$ & $317.0 \pm 7.7$ & $313.8 \pm 4.4$ & $301.8 \pm 6.5$ \\
e + p FAT & $5.7 \pm 0.2^{*}$ & $8.1 \pm 0.5$ & $7.4 \pm 0.6$ \\
Plasma triglyceride $(\mathrm{mg} / \mathrm{dl})$ & $48.0 \pm 1.1^{*}$ & $88.7 \pm 2.8$ & $83.6 \pm 6.5$ \\
Hepatic triglyceride $(\mathrm{mg} / \mathrm{g}$ tissue) & $14.5 \pm 1.1^{*}$ & $42.9 \pm 4.4$ & $34.7 \pm 3.0^{*}$ \\
& & &
\end{tabular}

${ }^{*} \mathrm{P}<0.05 ;-$, no fructose feeding. 

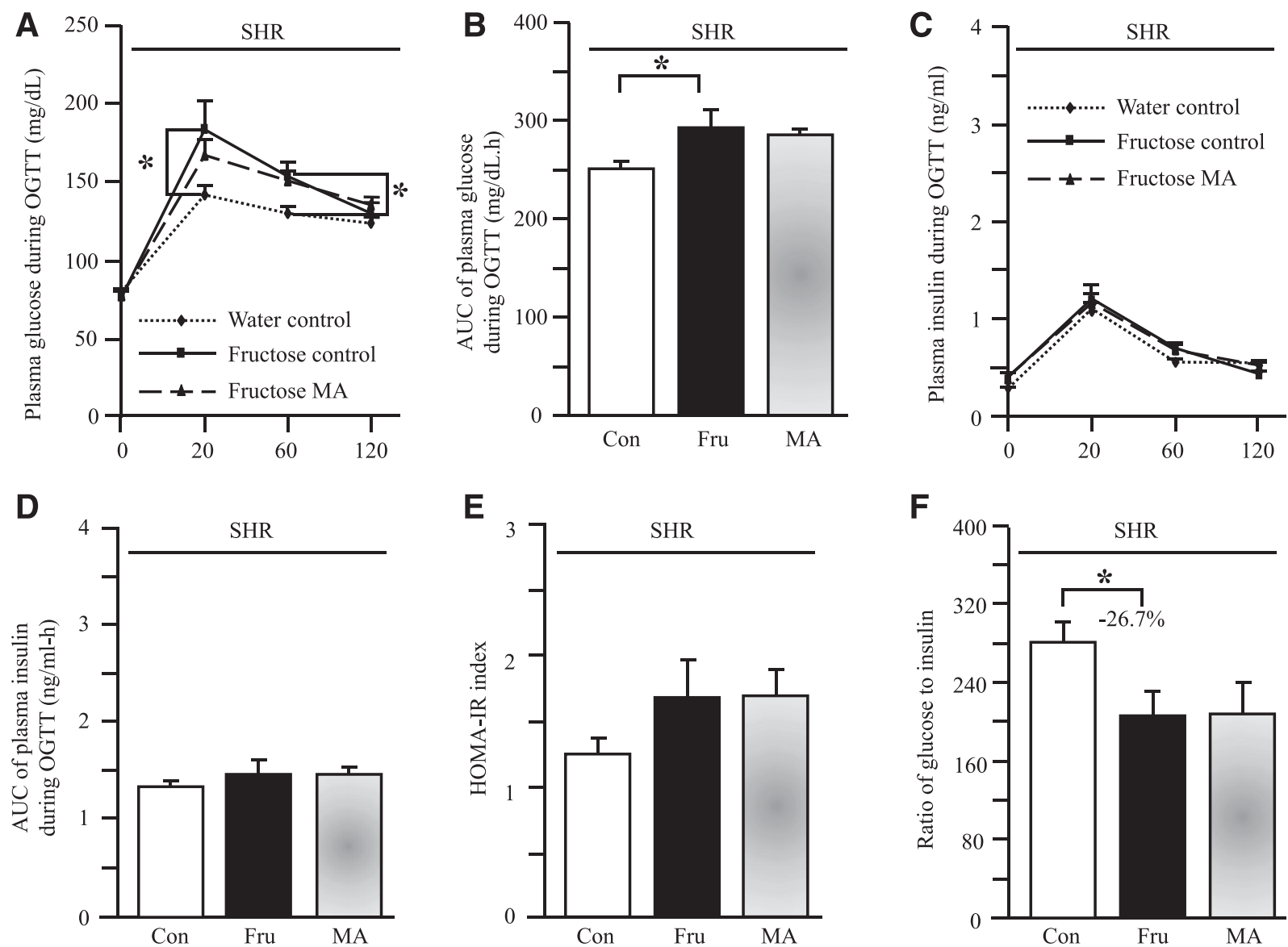

Fig. 6. Plasma glucose (A) and insulin (C) concentrations, and the AUC of glucose (B) and insulin (D) concentrations during OGTT, the HOMA-IR index (E), and the ratio of glucose to insulin (F) in SHR. The fructose controls (Fru) had free access to $10 \%$ fructose in their drinking water for 6 weeks, and the consumption of fructose in the mangiferin (MA) $(15 \mathrm{mg} / \mathrm{kg}$ )-treated (by gavage daily) rats was adjusted to match that of the fructose controls. The water controls (Con) had free access to a tap water. Data are means \pm S.E.M. $(n=6$ each group) versus control, $* P<0.05$.

skeletal muscle, thereby inhibiting glucose oxidation and reducing insulin sensitivity (Schwenk et al., 2008). Thus, abnormal fatty acid metabolism, especially in muscle, is linked to insulin resistance (Su and Abumrad, 2009). CD36 facilitates inward fatty acid transport, thus promoting the clearance of plasma fatty acids. In muscle from diabetic rodents and humans, more CD36 is recruited to the plasma membrane, leading to persistent enhancement of fatty acid uptake and contributing to impairment of insulin signaling and glucose utilization (Schwenk et al., 2008). In both obese animals and humans, the increased rates of long-chain fatty acid uptake across plasma membrane have been associated with increase in plasma membrane CD36, although total CD36 protein expression is not altered (Holloway et al., 2008). It has been demonstrated that CD36 expression is increased in the sarcolemma but not in whole skeletal muscle in fructose-fed rats, suggesting that a fructose-induced redistribution of this protein is associated with fatty acid uptake across the plasma membrane (Huynh et al., 2008). The present results showed that long-term fructose feeding increased triglyceride accumulation in the skeletal muscle of WKY. Although fructose did not alter CD36 mRNA and protein expression in whole-tissue homogenates, it resulted in sarcolemmal CD36 overexpression and less intracellular CD36 expression in the gastrocnemius. Mangiferin treatment tended to suppress the increase in triglyceride content in the skeletal muscle. Mangiferin did not affect CD36 mRNA and protein expression in the whole skeletal muscle, but it suppressed fructose-induced CD36 redistribution. These results suggest that mangiferin modulates fructose-induced CD36 redistribution in skeletal muscle, which may be associated with the suppression of fructose-induced acceleration in clearance of plasma NEFA during OGTT.

SHR carries a nonfunctional variant of the CD36 gene (Aitman et al., 1999). Although SHR had been demonstrated to express the CD36 mRNA and protein (Bonen et al., 2009), the CD36 functions may be disrupted. There are two different SHR strains, depending on the presence or absence of a de novo mutation in the gene for CD36 (Gotoda et al., 1999). The SHR/NCrl strain with a CD36 null mutation had reduced blood glucose and increased blood free fatty-acid levels compared with the SHR/Izm strain without this mutation (Gotoda et al., 1999). Furthermore, the SHR/ NCrl strain also showed decreased epididymal fat pad weight compared with the SHR/Izm strain (Gotoda et al., 1999). In the present study, we used the SHR/NCrl strain to confirm the involvement of CD36. In accordance with the reported phenotypes (Gotoda et al., 1999), our results showed that SHR at the baseline had decreased blood glucose concentration and epididymal fat pad weight, and increased blood free fatty acid level compared with WKY. Fructose-induced acceleration of clearance of plasma NEFA 

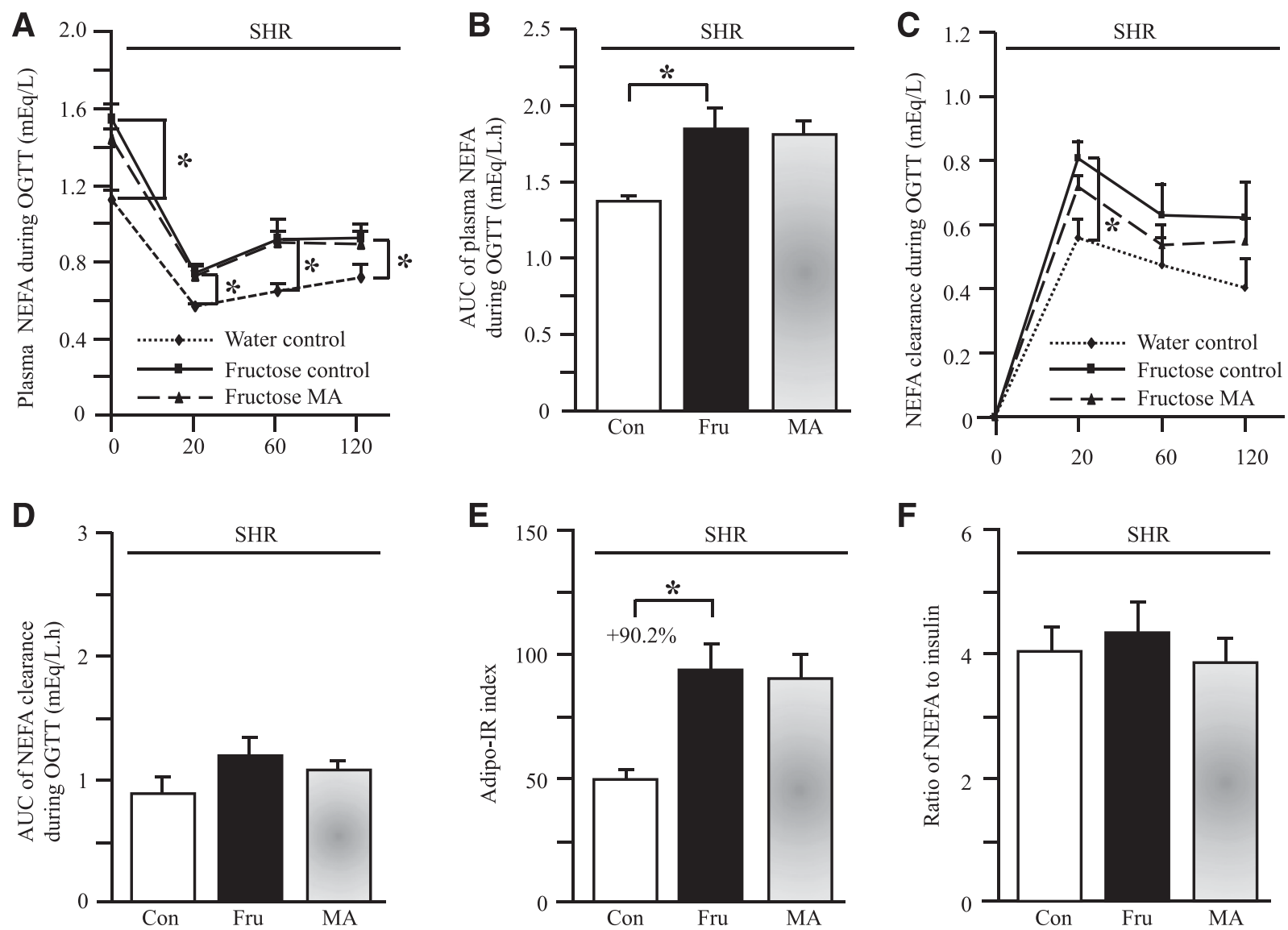

Fig. 7. Plasma NEFA concentrations (A), the AUC of plasma NEFA concentrations (B), plasma NEFA clearance (C), and the AUC of plasma NEFA clearance (D) during OGTT, the adipose tissue insulin resistance (Adipo-IR) index (E), and the ratio of NEFA to insulin (F) in SHR. The fructose controls (Fru) had free access to $10 \%$ fructose in their drinking water for 6 weeks, and the consumption of fructose in the mangiferin (MA) (15 mg/kg)-treated (by gavage daily) rats was adjusted to match that of the fructose controls. The water controls (Con) had free access to a tap water. Data are means \pm S.E.M. ( $n=6$ each group) versus control, ${ }^{*} P<0.05$.

during OGTT in WKY was attenuated in SHR. These results may support the deficiency of CD36 functions in SHR. On the other hand, fructose-induced increases in plasma insulin concentrations at the baseline and after oral glucose administration, in the HOMA-IR index, and in the Adipo-IR index, and a decrease in the ratio of glucose or NEFA to insulin were attenuated in SHR compared with those in WKY. These results may suggest a link between fructose-induced insulin resistance and CD36 functions. The striking effects elicited by mangiferin treatment on plasma insulin concentrations at the baseline and after oral glucose administration, on the HOMA-IR index and the Adipo-IR index, and on the ratio of glucose or NEFA to insulin in WKY were abolished in SHR. Moreover, mangiferin-induced suppression of fructoseinduced acceleration of plasma NEFA clearance during OGTT seen in WKY was also absent in SHR. These results may further support the association of modulation of CD36 redistribution with mangiferin-elicited insulin-sensitizing effect.

Mangiferin (50-150 mg/kg per day) has been demonstrated to have an effect on excessive hepatic triglyceride accumulation in high fat diet-fed hamsters (Guo et al., 2011) and Wistar rats (Niu et al., 2012), similar to that in WKY and SHR in the present study. However, the antisteatotic effect was accompanied by augmentation of high fat diet-stimulated overexpression of both hepatic and muscular CD36 mRNA in hamsters (Guo et al., 2011). It needs to be clarified whether the discrepancy is associated with the differences in models (high fat diet versus fructose), animal species (hamster versus WKY), dosages of mangiferin (50 and $150 \mathrm{mg} / \mathrm{kg}$ versus $15 \mathrm{mg} / \mathrm{kg}$ ), and/or other factors.

CD36 is a target of PPAR- $\gamma$ ligands; PPAR- $\gamma$ agonists stimulate the production of adiponectin, which promotes fatty acid oxidation and insulin sensitivity in muscle and liver (Tontonoz et al., 1998). It has been reported that mangiferin increased PPAR- $\gamma$ response element-driven luciferase activity in L6-myotubes (Girón et al., 2009). However, a gene reporter assay demonstrated that mangiferin did not affect ciglitazone-induced activation of all three isoforms of PPARs (PPAR- $\gamma$; PPAR- $\alpha$, PPAR- $\beta$ ) (Wilkinson et al., 2008). In contrast, mangiferin not only interacts with PPAR- $\gamma$ and enhances its DNA-binding ability but also suppresses phosphorylation and cytoplasmic translocation by modulating the mitogen-activated protein kinase/extracellular signalregulated kinase (MAPK-ERK) pathway in different cell lines (Mahali and Manna, 2012). These results suggest that mangiferin may be a modulator of PPAR- $\gamma$. Although the present results showed that mangiferin treatment did not alter muscular expression of PPAR- $\gamma$, adiponectin, MCP-1, and TNF- $\alpha$ in fructose-fed rats, further investigations are required to determine whether mangiferin modulates CD36 redistribution via the PPAR- $\gamma$ pathway. 
Although hepatic steatosis is strongly associated with the development of insulin resistance, it remains unclear whether insulin resistance causes the excessive accumulation of triglyceride in the liver, or whether the increase in triglyceride itself or of metabolite intermediates may play a causal role in the development of insulin resistance (Postic and Girard, 2008). Some studies have shown that the accumulation of intrahepatic lipids precedes the state of insulin resistance (Postic and Girard, 2008). It is feasible that amelioration of fructose-induced excessive hepatic triglyceride accumulation might contribute to the mitigation of insulin resistance in WKY. However, the antisteatotic effect of mangiferin was not accompanied by improvement of insulin resistance in SHR. Thus, these results seem to rule out the possibility that mangiferin improves insulin resistance secondary to the amelioration of hepatic steatosis in fructose-fed WKY.

In conclusion, our present results demonstrate that mangiferin mitigates insulin resistance in a rat model of fructoseinduced metabolic syndrome by modulation of sarcolemmal and intracellular CD36 redistribution in the skeletal muscle. Our study uncovers a novel mechanism of the antidiabetic activity of mangiferin. CD36 is expressed in a variety of cells, including monocytes, platelets, macrophages, microvascular endothelial cells, adipocytes, muscle cells, enterocytes, and hepatocytes; it can function in a range of processes unrelated to fatty acid uptake (e.g., binding of native and oxidized lipoproteins and signaling for apoptosis, phagocytosis, growth-hormone-releasing peptides, and Toll-like receptors) (Su and Abumrad, 2009). Mangiferin has documented antioxidant, cardioprotective, anti-inflammatory, and anticancer properties. Thus, CD36 would be useful in directing further study of the therapeutic effects of mangiferin and mangiferincontained herbs.

\section{Authorship Contributions}

Participated in research design: Zhou, Wang, Li.

Conducted experiments: Zhou, Chonan.

Performed data analysis: Zhou, Pan, Chonan, Batey, Rong, Yamahara, Wang, Li. $\mathrm{Li}$

Wrote or contributed to the writing of the manuscript: Zhou, Wang,

\section{References}

Aitman TJ, Glazier AM, Wallace CA, Cooper LD, Norsworthy PJ, Wahid FN, AlMajali KM, Trembling PM, Mann CJ, and Shoulders CC, et al. (1999) Identification of Cd36 (Fat) as an insulin-resistance gene causing defective fatty acid and glucose metabolism in hypertensive rats. Nat Genet 21:76-83.

Akase T, Shimada T, Harasawa Y, Akase T, Ikeya Y, Nagai E, Iizuka S, Nakagami G, Iizaka S, and Sanada H, et al. (2011) Preventive Effects of Salacia reticulata on Obesity and Metabolic Disorders in TSOD Mice. Evid Based Complement Alternat Med 2011:484590.

Apontes P, Liu Z, Su K, Benard O, Youn DY, Li X, Li W, Mirza RH, Bastie CC and Jelicks LA, et al. (2014) Mangiferin stimulates carbohydrate oxidation and protects against metabolic disorders induced by high-fat diets. Diabetes 63: $3626-3636$

Bonen A, Han XX, Tandon NN, Glatz JFC, Lally J, Snook LA, and Luiken JJFP (2009) FAT/CD36 expression is not ablated in spontaneously hypertensive rats. $J$ Lipid Res 50:740-748.

Cornier MA, Dabelea D, Hernandez TL, Lindstrom RC, Steig AJ, Stob NR, Van Pelt RE, Wang H, and Eckel RH (2008) The metabolic syndrome. Endocr Rev 29 $777-822$.

Eckel RH, Grundy SM, and Zimmet PZ (2005) The metabolic syndrome. Lancet 365: $1415-1428$.

Eckel RH, Alberti KG, Grundy SM, and Zimmet PZ (2010) The metabolic syndrome. Lancet 375:181-183.

Ferrannini E, Buzzigoli G, Bonadonna R, Giorico MA, Oleggini M, Graziadei L, Pedrinelli R, Brandi L, and Bevilacqua S (1987) Insulin resistance in essentia hypertension. N Engl J Med 317:350-357.

Gastaldelli A, Harrison SA, Belfort-Aguilar R, Hardies LJ, Balas B, Schenker S, and Cusi K (2009) Importance of changes in adipose tissue insulin resistance to histological response during thiazolidinedione treatment of patients with nonalcoholic steatohepatitis. Hepatology 50:1087-1093.
Girón MD, Sevillano N, Salto R, Haidour A, Manzano M, Jiménez ML, Rueda R, and López-Pedrosa JM (2009) Salacia oblonga extract increases glucose transporter 4-mediated glucose uptake in L6 rat myotubes: role of mangiferin. Clin Nutr 28:565-574.

Gotoda T, Iizuka Y, Kato N, Osuga J, Bihoreau MT, Murakami T, Yamori Y, Shimano H, Ishibashi S, and Yamada N (1999) Absence of Cd36 mutation in the original spontaneously hypertensive rats with insulin resistance. Nat Genet $\mathbf{2 2}$ $226-228$

Guo F, Huang C, Liao X, Wang Y, He Y, Feng R, Li Y, and Sun C (2011) Beneficial effects of mangiferin on hyperlipidemia in high-fat-fed hamsters. Mol Nutr Food Res 55:1809-1818.

Hajri T, Ibrahimi A, Coburn CT, Knapp FF, Jr, Kurtz T, Pravenec M, and Abumrad NA (2001) Defective fatty acid uptake in the spontaneously hypertensive rat is a primary determinant of altered glucose metabolism, hyperinsulinemia, and myocardial hypertrophy. J Biol Chem 276:23661-23666.

Han J, Yang N, Zhang F, Zhang C, Liang F, Xie W, and Chen W (2015) Rhizoma Anemarrhenae extract ameliorates hyperglycemia and insulin resistance via activation of AMP-activated protein kinase in diabetic rodents. J Ethnopharmacol 172:368-376.

Hanson RL, Imperatore G, Bennett PH, and Knowler WC (2002) Components of the "metabolic syndrome" and incidence of type 2 diabetes. Diabetes 51:3120-3127.

Holloway GP, Luiken JJ, Glatz JF, Spriet LL, and Bonen A (2008) Contribution of FAT/CD36 to the regulation of skeletal muscle fatty acid oxidation: an overview. Acta Physiol (Oxf) 194:293-309.

Huang TH, Peng G, Li GQ, Yamahara J, Roufogalis BD, and Li Y (2006a) Salacia oblonga root improves postprandial hyperlipidemia and hepatic steatosis in Zucker diabetic fatty rats: activation of PPAR-alpha. Toxicol Appl Pharmacol 210: $225-235$

Huang TH, Yang Q, Harada M, Uberai J, Radford J, Li GQ, Yamahara J, Roufogalis $\mathrm{BD}$, and $\mathrm{Li} \mathrm{Y}$ (2006b) Salacia oblonga root improves cardiac lipid metabolism in Zucker diabetic fatty rats: modulation of cardiac PPARalpha-mediated transcription of fatty acid metabolic genes. Toxicol Appl Pharmacol 210:78-85.

Huynh M, Luiken JJ, Coumans W, and Bell RC (2008) Dietary fructose during the suckling period increases body weight and fatty acid uptake into skeletal muscle in adult rats. Obesity (Silver Spring) 16:1755-1762.

Ichiki H, Miura T, Kubo M, Ishihara E, Komatsu Y, Tanigawa K, and Okada M (1998) New antidiabetic compounds, mangiferin and its glucoside. Biol Pharm Bull 21:1389-1390.

Kelley DE, Goodpaster BH, and Storlien L (2002) Muscle triglyceride and insulin resistance. Annu Rev Nutr 22:325-346.

Lebovitz HE (1999) Type 2 diabetes: an overview. Clin Chem 45:1339-1345.

Lewis GF, Carpentier A, Adeli K, and Giacca A (2002) Disordered fat storage and mobilization in the pathogenesis of insulin resistance and type 2 diabetes. Endocr Rev 23:201-229.

Li Y, Huang TH, and Yamahara J (2008) Salacia root, a unique Ayurvedic medicine, meets multiple targets in diabetes and obesity. Life Sci 82:1045-1049.

Li Y, Peng G, Li Q, Wen S, Huang TH, Roufogalis BD, and Yamahara J (2004) Salacia oblonga improves cardiac fibrosis and inhibits postprandial hyperglycemia in obese Zucker rats. Life Sci 75:1735-1746.

Liu L, Yang M, Lin X, Li Y, Liu C, Yang Y, Yamahara J, Wang J, and Li Y (2013) Modulation of hepatic sterol regulatory element-binding protein-1c-mediated gene expression contributes to Salacia oblonga root-elicited improvement of fructoseinduced fatty liver in rats. J Ethnopharmacol 150:1045-1052.

Love-Gregory L and Abumrad NA (2011) CD36 genetics and the metabolic complications of obesity. Curr Opin Clin Nutr Metab Care 14:527-534.

Love-Gregory L, Sherva R, Schappe T, Qi JS, McCrea J, Klein S, Connelly MA and Abumrad NA (2011) Common CD36 SNPs reduce protein expression and may contribute to a protective atherogenic profile. Hum Mol Genet 20:193-201.

Machann J, Häring H, Schick F, and Stumvoll M (2004) Intramyocellular lipids and insulin resistance. Diabetes Obes Metab 6:239-248.

Mahali SK and Manna SK (2012) Beta-D-glucoside protects against advanced glycation end products (AGEs)-mediated diabetic responses by suppressing ERK and inducing PPAR gamma DNA binding. Biochem Pharmacol 84:1681-1690.

Muruganandan S, Srinivasan K, Gupta S, Gupta PK, and Lal J (2005) Effect of mangiferin on hyperglycemia and atherogenicity in streptozotocin diabetic rats. $J$ Ethnopharmacol 97:497-501.

Na L, Zhang Q, Jiang S, Du S, Zhang W, Li Y, Sun C, and Niu Y (2015) Mangiferin supplementation improves serum lipid profiles in overweight patients with hyperlipidemia: a double-blind randomized controlled trial. Sci Rep 5:10344.

Neuschwander-Tetri BA (2010) Hepatic lipotoxicity and the pathogenesis of nonalcoholic steatohepatitis: the central role of nontriglyceride fatty acid metabolites. Hepatology 52:774-788.

Niu Y, Li S, Na L, Feng R, Liu L, Li Y, and Sun C (2012) Mangiferin decreases plasma free fatty acids through promoting its catabolism in liver by activation of AMPK. PLoS One 7:e30782.

Postic C and Girard J (2008) Contribution of de novo fatty acid synthesis to hepatic steatosis and insulin resistance: lessons from genetically engineered mice. J Clin Invest 118:829-838.

Schwenk RW, Luiken JJ, Bonen A, and Glatz JF (2008) Regulation of sarcolemmal glucose and fatty acid transporters in cardiac disease. Cardiovasc Res 79:249-258. Stanhope KL, Schwarz JM, Keim NL, Griffen SC, Bremer AA, Graham JL, Hatcher B, Cox CL, Dyachenko A, and Zhang W, et al. (2009) Consuming fructosesweetened, not glucose-sweetened, beverages increases visceral adiposity and lipids and decreases insulin sensitivity in overweight/obese humans. $J$ Clin Invest 119:1322-1334.

Su X and Abumrad NA (2009) Cellular fatty acid uptake: a pathway under construction. Trends Endocrinol Metab 20:72-77.

Tappy L and Lê KA (2010) Metabolic effects of fructose and the worldwide increase in obesity. Physiol Rev 90:23-46. 
Tontonoz P, Nagy L, Alvarez JG, Thomazy VA, and Evans RM (1998) PPARgamma promotes monocyte/macrophage differentiation and uptake of oxidized LDL. Cell 93:241-252.

Vistisen B, Roepstorff K, Roepstorff C, Bonen A, van Deurs B, and Kiens B (2004) Sarcolemmal FAT/CD36 in human skeletal muscle colocalizes with caveolin-3 and is more abundant in type 1 than in type 2 fibers. J Lipid Res 45:603-609.

Wilkinson AS, Monteith GR, Shaw PN, Lin CN, Gidley MJ, and Roberts-Thomson SJ (2008) Effects of the mango components mangiferin and quercetin and the putative mangiferin metabolite norathyriol on the transactivation of peroxisome proliferator-activated receptor isoforms. J Agric Food Chem 56:3037-3042.

Xing X, Li D, Chen D, Zhou L, Chonan R, Yamahara J, Wang J, and Li Y (2014)

Mangiferin treatment inhibits hepatic expression of acyl-coenzyme A:diacylglycerol acyltransferase- 2 in fructose-fed spontaneously hypertensive rats: a link to amelioration of fatty liver. Toxicol Appl Pharmacol 280:207-215.

Address correspondence to: Dr. Jianwei Wang, The Laboratory of Traditional Chinese Medicine, Chongqing Medical University, 1 Yixueyuan Road, Yuzhong District, Chongqing 400016, China. E-mail: wjwcq68@163.com; or Yuhao Li, Endocrinology and Metabolism Group, Sydney Institute of Health Sciences/Sydney Institute of Traditional Chinese Medicine, Level 5, 545 Kent Street, Sydney NSW 2000 Australia. E-mail: yuhao@sitcm.edu.au 